\title{
INTERPRETIVE SUMMARIES, FEBRUARY 2013
}

Metabolism of milk fat globule membrane components by nonstarter lactic acid bacteria isolated from cheese. By Moe et al., page 727. This study was conducted to gain insight into the importance of the milk fat-associated components in cheese for the development of bacteria involved in the cheese-ripening process. Cheese-ripening bacteria were grown in different media containing components associated with the milk fat in cheese, and the degradation of these components was monitored. Cheese-ripening bacteria were able to utilize sugars associated with the milk fat in cheese for growth and survival, and the metabolites produced differed depending on which components the bacteria utilized.

Automatic milking systems in the Protected Designation of Origin Montasio cheese production chain: Effects on milk and cheese quality. By Innocente and Biasutti, page 740. The objective of this study was to evaluate the effects of variations in milking frequency associated with the adoption of an automatic milking system on milk quality and on the characteristics of Protected Designation of Origin Montasio cheese. This cheese's distinctive characteristics derive from the traditional production methods involved (milk production, manufacturing techniques, and ripening process). Thus, it is particularly important to evaluate the effect of a technological innovation in milk production on those milk quality features that may affect the cheese-making process and on the chemical indices associated with cheese quality

Molecular screening of ovine mastitis in different breeds. By Guerreiro et al., page 752. A novel molecular screening method, based on an optimized raw milk DNA extraction, was developed to identify pathogens such as Staphylococcus aureus, Streptococcus agalactiae, Streptococcus dysgalactiae, and Streptococcus uberis. The molecular method was a faster and more precise alternative to current diagnostic methods, allowing more efficient animal treatment and leading to less expensive management of dairy flocks.

A comprehensive dairy valorization model. $B y$ Banaszewska et al., page 761. This paper presents a comprehensive dairy valorization model that serves as a decision support tool for dairy processors. The model was designed to create mid-term plans for the allocation of milk and the production of end products. Raw milk was allocated to the most profitable dairy products while taking important constraints into account; that is, recipes, variations in composition, dairy production interdependencies, seasonality, demand, supply, capacities, and transportation flows. The model was tested at the international dairy processor FrieslandCampina. To illustrate the functionality of the model, we analyzed in detail the effect of seasonality on the valorization of milk.

Effects of different fermentation parameters on quality characteristics of kefir. By Kök Tass et al., page 780. The main objective of this study was to determine the effects of different fermentation parameters on kefir quality. We present significant findings with regard to the evaluation of probiotic content and microflora of kefir, which are arguably the most important criteria for its therapeutic efficacy. These findings are a significant contribution to the literature and to the dairy industry.

Binding of vitamin A by casein micelles in commercial skim milk. By Mohan et al., page 790. Vitamin A, a fat-soluble vitamin present naturally in whole milk, is fortified in skim milk to compensate for the loss on removal of milk fat and to meet daily requirements in the diet. Caseins that form a major portion of the milk proteins (80\%) exist as micelles in milk, having channels and cavities within its structure and held together by hydrophobic interactions. We studied the ability of casein micelles to entrap vitamin A and found that up to $40 \%$ of the vitamin A added to skim milk was associated with the casein micelles.

Multiple-dose therapy with bovine colostrum confers significant protection against diarrhea in a mouse model of human rotavirus-induced gastrointestinal disease. By Inagaki et al., page 806. This work showed that multiple doses of bovine late colostrum, obtained from healthy lactating cows on d 6 to 7 after parturition, relieved diarrheal symptoms caused by human rotavirus in suckling mice. Bovine late colostrum, which contains abundant immunoglobulin $\mathrm{G}$, is a promising natural candidate for routine management of human rotavirus infection.

Fate of Shiga toxin-producing and generic Escherichia coli during production and ripening of semihard raw milk cheese. By Peng et al., page 815 . Shiga toxin-producing Escherichia coli (STEC) is an important foodborne pathogen able to cause severe disease in humans. Raw milk cheeses have the potential to carry STEC. Therefore, we investigated the ability of STEC and generic E. coli to survive the production and ripening of raw milk cheese. During production, a strong increase was observed for the strains tested, whereas during ripening, a slow continuous decrease occurred . However, STEC were detected in most cheeses at the end of the ripening period. This finding is a matter of concern because low numbers of STEC may be sufficient to cause disease in humans. 
Effect of calcium in brine on salt diffusion and water distribution of Mozzarella cheese during brining. By Luo et al., page 824. Mozzarella is typically salted in brine, which is critical for proper moisture control and for its textural, physical, and flavor properties. However, a soft, pasty, high-moisture surface called a soft surface defect often occurs during brining. The traditional solution is to add calcium to the brine. The objective of the present study was to examine the mechanism underlying the effect of calcium in brine on Mozzarella cheese during brining. A detailed understanding of the mechanism would enable operators to optimize the process and thus improve control of the final product.

Effect of Bifidobacterium bifidum BF-1 on gastric protection and mucin production in an acute gastric injury rat model. By Gomi et al., page 832. Fermented milk containing the probiotic bacterium $B i$ fidobacterium bifidum $\mathrm{BF}-1$ (BF-1) is known to improve Helicobacter pylori-associated gastritis in humans. In this study, we investigated the gastroprotective effects of BF-1 in a rat model of acute gastric injury. Following acid-ethanol treatment to induce acute gastric injury, the injury rate was significantly lower in rats that had been fed BF-1 than in control rats fed vehicle only. Interestingly, gastric mucin production in the stomach was significantly enhanced in the BF-1 group compared with controls. These findings indicate that BF-1 has the potential to alleviate acute gastric injury via enhancement of gastric mucin production.

Angiotensin I-converting enzyme inhibitory activity of the Norwegian autochthonous cheeses Gamalost and Norvegia after in vitro human gastrointestinal digestion. By Qureshi et al., page 838. Gamalost, a traditional Norwegian cheese known to have very high angiotensin I-converting enzyme (ACE) inhibitory activity (i.e., antihypertensive effect), was digested by human gastric juice and human duodenal juice at $37^{\circ} \mathrm{C}$ in an incubator, mimicking conditions in the human body. We found that ACE-inhibitory activity was slightly increased after digestion due to the release of many potential ACE-inhibitory peptides from the cheese protein. Norvegia, a Gouda-type cheese, had much lower ACE-inhibitory activity than Gamalost; however, during digestion, its ACE-inhibitory activity increased almost to the level of Gamalost.

Hot topic: Comparison of sex-sorted and conventional semen within a fixed-time artifical insemination protocol designed for dairy heifers. By Mallory et al., page 854. The objective was to compare pregnancy per artificial insemination (P/AI) in dairy heifers inseminated with conventional and sexsorted semen within a fixed-time AI (FTAI) program (Show-Me-Synch). Pregnancy per AI resulting from
FTAI was greater for heifers that were inseminated with conventional semen compared with those that received sex-sorted semen. The expression of estrus before FTAI did not affect $\mathrm{P} / \mathrm{AI}$ when conventional semen was used, whereas $\mathrm{P} / \mathrm{AI}$ with sex-sorted semen was greater for heifers detected in estrus. Further studies are required to develop strategies for using sex-sorted semen when inseminating heifers at predetermined fixed times on the basis of expression of estrus before FTAI.

The carbon footprint of pasture-based milk production: Can white clover make a difference? By Yan et al., page 85\%. We found that substituting fertilizer $\mathrm{N}$ with white clover in pasture reduced the greenhouse gas emissions of milk production by 11 to $23 \%$. The modeled $\mathrm{N}_{2} \mathrm{O}$ emissions were close to the measured $\mathrm{N}_{2} \mathrm{O}$ emissions from both swards under similar conditions. Sensitivity analysis showed that the difference between the 2 systems was robust toward modeling assumptions. We concluded that introducing clover could reduce the carbon footprint of milk from grazing systems.

Postoperative analgesic efficacy of meloxicam in lame dairy cows undergoing resection of the distal interphalangeal joint. By Offinger et al., page 866. This study investigated benefits and adverse reactions of repeated doses of the analgesic meloxicam in a standard anesthesia protocol in cows undergoing radical claw surgery. A reduction in plasma cortisol levels and heart rate indicated the analgesic properties of meloxicam in the postsurgical period. This was accompanied by reduced lameness scores, increased standing periods, and higher activity in meloxicam-treated cows. No indications for gastrointestinal bleeding were recorded. In conclusion, repeated meloxicam application demonstrated effective analgesia in the postsurgical period after extensive claw surgery in dairy cows without evidence for adverse effects on gastrointestinal integrity.

Concomitant lipopolysaccharide-induced transfer of blood-derived components including immunoglobulins into milk. By Lehmann et al., page 889. By the use of animals with manipulated blood $\beta$-hydroxybutyrate (BHBA) concentrations, this study confirmed the expected blood origin of a number of factors that increase in milk during the early phase of lipopolysaccharide (LPS)-induced mastitis and described the time course of their increase relative to that of somatic cell count. Milk concentrations of L-lactate, BHBA, lactate dehydrogenase, immunoglobulin G1, and immunoglobulin G2 increased concomitantly in LPS-challenged quarters, suggesting their blood origin. Many of these blood components leak into milk but are not necessarily involved in mammary immune defense. 
Variation in milk cortisol during lactation in Murciano-Granadina goats. By Díaz et al., page 897. Determination of milk cortisol concentration can be an appropriate noninvasive method to assess the welfare of dairy cattle. The purpose of this work was to analyze how certain factors, such as characteristics associated with the animal, automatic milking, and environmental conditions, affect concentrations of cortisol in goat milk. This would enable us to recognize when increases of cortisol in milk are caused by stressful factors. We concluded that cortisol milk concentration can change depending on certain physiological conditions, such as milk production level, parity number, and lactation stage.

Economics of resynchronization strategies including chemical tests to identify nonpregnant cows. By Giordano et al., page 949. The potential economic value of chemical tests for nonpregnancy diagnosis may be due to earlier identification of nonpregnant cows, which, when coupled with a resynchronization strategy, reduces the interval between inseminations, thereby increasing profitability. Simulation experiments showed that performing a chemical test $8 \mathrm{~d}$ earlier than rectal palpation or transrectal ultrasound to identify nonpregnant cows resulted in both greater and lesser economic benefits, depending on the potential negative effect of inaccuracy of the chemical test, pregnancy loss, and the cost of the test. The major effect of using a chemical test was the potential to decrease the interbreeding interval.

Comparative molecular analysis of ovine and bovine Streptococcus uberis isolates. By Gilchrist et al., page 962. Streptococcus uberis is an important mastitis-causing pathogen of dairy cattle. It may also cause subclinical and clinical mastitis in sheep. Comparative molecular analysis of Strep. uberis from milk showed that ovine isolates are distinct from bovine isolates, and more likely than bovine isolates to carry the alternative plasminogen activator pauB. Ovine Strep. uberis is a potential source of genetic material that could affect the efficacy of future bovine mastitis vaccines if those were developed without consideration of the full spectrum of Strep. uberis heterogeneity. Furthermore, our results indicate that lateral gene transfer between streptococcal species contributes to Strep. uberis heterogeneity.

Short-term feed intake is regulated by macronutrient oxidation in lactating Holstein cows. By Derno et al., page 971. Among several endogenous signals, temporal patterns of metabolic oxidative processes are thought to be involved in short-term feed intake regulation in dairy cows. We aimed to elucidate how the extent of periprandial net carbohydrate oxidation $\left(\mathrm{COX}_{\text {net }}\right)$ and net fat oxidation $\left(\mathrm{FOX}_{\text {net }}\right)$ inte- grate ad libitum feed intake and corresponding plasma metabolites and hormone concentrations. Feed intake events were strongly cross-correlated with $\mathrm{COX}_{\text {net }}$ and $\mathrm{FOX}_{\text {net }}$, suggesting that cows began feeding when the FOX $_{\text {net }}$ rate exceeded $-0.2 \mathrm{~g} / \mathrm{min}$ and the $\mathrm{COX}_{\text {net }}$ rate decreased below $6 \mathrm{~g} / \mathrm{min}$.

First-calving age and first-lactation milk production on Dutch dairy farms. By Mohd Nor et al., page 981. Data of 8,545 heifers from 100 Dutch dairy farms were used to determine the association between first-calving age and milk production in the first lactation. Calving 1 mo earlier than the median first-calving age of the herd resulted in less milk $(90 \mathrm{~kg} / 305 \mathrm{~d})$ in the first lactation. This indicates that earlier insemination without adjusting management to ensure sufficient development lowers milk production. As a consequence, decisions with regard to management of young stock should made with care.

Bovine Staphylococcus aureus: Dose response to iodine and chlorhexidine and effect of iodine challenge on antibiotic susceptibility. By Azizoglu et al., page 993. The use of germicidal teat dips is one of the measures taken by the dairy industry to control mastitis. Iodine and chlorhexidine compounds are commonly used disinfectants in teat dips. In this study, we evaluated the minimum inhibitory concentration (MIC) of iodine for a major mastitis-causing pathogen, Staphylococcus aureus. In addition, we determined iodine and chlorhexidine dose responses of selected isolates of Staph. aureus. Differences between Staph. aureus isolates in MIC of iodine and dose response against iodine were observed. All isolates tested were susceptible to chlorhexidine.

Evaluation of presynchronized resynchronization protocols for lactating dairy cows. By Chebel et al., page 1009. Approximately $60 \%$ of lactating dairy cows do not conceive to first postpartum insemination and need to have their estrous cycle resynchronized for timely re-insemination following nonpregnancy diagnosis. In the current experiment, presynchronization with gonadotropin-releasing hormone and prostaglandin $\mathrm{F}_{2 \alpha} 7$ and $11 \mathrm{~d}$, respectively, before the start of the resynchronization protocol resulted in similar overall pregnancy per re-insemination, but cows presynchronized with prostaglandin $\mathrm{F}_{2 \alpha}$ were re-inseminated at a faster rate and had greater pregnancy rate within 7 and $14 \mathrm{~d}$ after nonpregnancy diagnosis. Treatment with a controlled internal drug release insert during the resynchronization protocol, however, did not affect pregnancy to timed re-insemination.

Relationship among specific bacterial counts and total bacterial and somatic cell counts and factors influencing their variation in ovine bulk 
tank milk. By Garnica et al., page 1021. Bulk tank total bacterial count (BTTBC) and somatic cell count (BTSCC) variables, as determined in dairy laboratories, are useful variables for monitoring milk hygiene quality in dairy sheep. Psychrotrophic, coliform, and thermoduric bacteria were significantly related to BTTBC, whereas gram-positive, catalase-negative cocci were correlated with both BTTBC and BTSCC variables in dairy sheep flocks. Season significantly affected all specific bacterial counts of milk.

Cow-level association between serum 25-hydroxyvitamin D concentration and Mycobacterium avium subspecies paratuberculosis antibody seropositivity: A pilot study. By Sorge et al., page 1030. Vitamin D plays an integral part in bone metabolism and immune function. Its deficiency has been associated with diseases in humans. Johne's disease is an economically important disease of ruminants. This study investigated the hypothesis that cows with Mycobacterium avium ssp. paratuberculosis (MAP) antibodies have lower serum 25-hydroxyvitamin D levels compared with MAP-antibody negative herdmates. Although cows positive for MAP antibodies had lower vitamin D levels compared with test-negative herdmates, the mean 25-hydroxyvitamin D level of study cows was $62.5 \mathrm{ng} / \mathrm{mL}$. Overall, the cows were fed vitamin D at very high levels, so additional supplementation with vitamin $\mathrm{D}$ would likely have little effect.

Expression of target genes of nuclear factor E2related factor 2 in the liver of dairy cows in the transition period and at different stages of lactation. By Gessner et al., page 1038. In the liver of dairy cows, the production of cytokines is enhanced during the periparturient phase, which in turn leads to inflammation and impairment of hepatic function. Nuclear factor E2-related factor 2 (Nrf2) is a redox-sensitive transcription factor that controls the transcription of various antioxidative and cytoprotective proteins. In the present study, we observed that many Nrf2 target genes with antioxidative and cytoprotective functions were strongly upregulated during the transition from pregnancy to lactation. The upregulation of these genes could represent a response to mitigate the damaging effects of increased concentrations of cytokines and reactive oxygen species in the liver of dairy cows during early lactation.

Evaluation of a clay-based acidic bedding conditioner for dairy cattle bedding. By Proietto et al., page 1044. Mastitis is one of the most prevalent diseases facing the dairy industry today. Control measures are now being implemented for contagious mastitis pathogens, but environmental mastitis pathogens remain a problem. Environmental pathogens reside in the cow's environment such as alley ways and bedding material. A clay-based acidic bedding conditioner added to sawdust was found to reduce the $\mathrm{pH}$ of the total bedding material, environmental mastitis pathogens in the bedding, and environmental mastitis pathogens on the teat ends of mid-lactation Holstein cows. In addition, the acidic bedding conditioner did not affect teat end integrity.

Determination of ceftiofur derivatives in serum, endometrial tissue, and lochia in puerperal dairy cows with acute puerperal metritis after subcutaneous administration of ceftiofur crystalline free acid. By von Krueger et al., page 1054. Feverish puerperal uterine infections such as metritis cause decreased reproductive performance. The treatment of choice is systemic administration of antibiotics to reduce the effect of uterine infections on fertility. Efficacy of an antibiotic requires concentrations above the minimum inhibitory concentration of relevant pathogens. Our results demonstrate that, after a single subcutaneous administration of ceftiofur crystalline free acid at the base of the ear, concentrations of ceftiofur derivatives in serum of dairy cows with feverish metritis remained above the reported minimum inhibitory concentration of relevant pathogens 4 to $6 \mathrm{~d}$ after injection. The proportion of cows with concentrations of ceftiofur derivatives in endometrial tissue and lochia above the minimum inhibitory concentration 4 to $6 \mathrm{~d}$ after injection was low.

Evaluation of transrectal examination of the cervical diameter by palpation in dairy cows. By Leutert et al., page 1063. Delayed involution of the cervix has been described as a distinct manifestation of uterine disease. Currently, only limited data are available on the reliability of assessment of cervical diameter by transrectal palpation. We demonstrated that estimates of cervical diameter obtained through transrectal palpation show high variation, low sensitivity, and only moderate correlation with ultrasound measurements as the reference standard. Variability increased with larger diameter, but results can be improved with training.

Short communication: Effects of milking frequency on udder morphology, milk partitioning, and milk quality in 3 dairy goat breeds. By Torres et al., page 1071. Some dairy goat breeds are milked once a day, which is less expensive, reduces the workload of farmers, and improves their living standards. On the other hand, in zones where high-yielding dairy goats are used for commercial purposes, milking is usually done twice a day. Here, we report the effects of unilateral milking frequency on udder morphological parameters, milk partitioning, and somatic cell count in 3 dairy goat breeds. We concluded that greater milk transfer from alveoli to cistern takes place in early udder filling. 
Short communication: Associations between teat dimensions and milking-induced changes in teat dimensions, and quarter milk somatic cell counts in dairy cows. By Zwertvaegher et al., page 1075. Quarter milk somatic cell count (SCC), and pre- and postmilking teat length and teat diameter of 72 dairy cows were determined in a longitudinal study. Both of the premilking measures (as relative changes of the teat barrel diameter) were significantly associated with quarter milk SCC, indicating that these teat dimensions could be useful to improve milk quality and udder health, either by directed (genetic) selection towards more optimal teat characteristics, or as indicators for improper milking and incorrect machine settings, including evaluation of the teatcup liner.

Short communication: Ability of dogs to detect estrus from sniffing saliva samples. By FischerTenhagen et al., page 1081. Estrus detection is still a major challenge in bovine reproduction. In this study, we tested the ability of dogs to detect estrus-specific smells in saliva of cows. We demonstrated that dogs could discriminate between saliva samples from cows in estrus and in diestrus but that overall accuracy was insufficient.

Short communication: Acute but transient increase in serum insulin reduces messenger RNA expression of hepatic enzymes associated with progesterone catabolism in dairy cows. By Vieira et al., page 1085. In cows, insulin modulates circulating concentrations of progesterone, a hormone required for optimal fertility and pregnancy maintenance. Furthermore, circulating concentrations of progesterone and insulin are modulated by cow nutrient intake. Herein, intravenous glucose infusion acutely and transiently increased circulating insulin concentrations and promptly reduced hepatic gene expression of CYP2C19 and CYP3A4, 2 enzymes associated with progesterone catabolism. Given the importance of progesterone to reproduction, this research provides novel and noteworthy insight on the physiological mechanisms associating nutrition and reproductive function in dairy cows.

Technical note: Monitoring grazing bites and walking activity with pedometers. By Umemura, page 1090. Three types of pedometers were attached to the neck collars of dairy cows to record the number of grazing bites. The values recorded by the pedometers were linearly related to the number of bites measured by visual observation and were not affected by rumination. The number of back-and-forth movements was greater during grazing than during walking, whereas right-toleft movement values were similar during grazing and walking. These differences will aid in distinguishing grazing and walking behaviors.
Effects of short-chain fructooligosaccharides on growth performance of preruminant veal calves. By Grans et al., page 1094. Improvement of growth performance in livestock is important for farmers. It may be enhanced by inclusion of prebiotic-like shortchain fructooligosaccharides. The effects of short-chain fructooligosaccharides have been poorly investigated in veal calves. In the present study, the inclusion of this prebiotic ingredient in calf milk replacer increased growth performance of preruminant calves, as demonstrated by the increase in carcass weight and specific indices characterizing animals' growth performance.

Effect of rumen-protected choline supplementation on liver and adipose gene expression during the transition period in dairy cattle. By Goselink et al., page 1102. In dairy cattle, the start of lactation stimulates the mobilization of adipose tissue, resulting in an increased concentration of triacylglycerol in the liver. We previously showed that rumen-protected choline supplementation reduced hepatic triacylglycerol accumulation after calving, thus improving animal health and production. The mode of action of choline was further investigated by analyzing the messenger RNA expression levels of key enzymes in liver and adipose tissue metabolism and the fat composition of the milk. Rumen-protected choline does not affect adipose mobilization but has an important role through improving fatty acid binding, transport, and secretion at the liver.

Effect of physical form of forage on performance, feeding behavior, and digestibility of Holstein calves. By Montoro et al., page 111\%. The physical form of forage may influence rumen development, and consequently body weight gain, dry matter consumption, digestibility, and welfare of dairy calves. The aim of this study was to determine the effect of 2 different physical forms of forage (chopped or ground) on performance, apparent digestibility, and feeding behavior of young calves. We concluded that providing chopped hay to young calves improves feed intake and nutrient digestibility during the week after weaning and reduces nonnutritive oral behaviors compared with offering ground hay.

Effects of milk replacer program fed 2 or 4 times daily on nutrient intake and calf growth. By Kmicikewycz et al., page 1125. The effects of milk replacer (MR) and MR feeding frequency $(\mathrm{FF})$ on nursery calf growth, growth efficiency, and starter intake was investigated. Calves were fed, in 2 or 4 meals/d, a 20:20 (protein:fat) MR at 1.5\% of body weight (STD2 and STD4) or a 26:18 MR at 2.0\% of body weight (MOD2 and MOD4). Calves fed MOD had higher intakes of crude protein and fat, greater average daily gain, greater gain:feed, and lower starter intake but 
not dry matter intake compared with calves fed STD. Feeding frequency had no effect on growth, starter intake, or gain:feed. Greater FF increased 56-d starter intake, heart girth, and serum nonesterified fatty acids in calves fed STD but not MOD. By d 56, calves fed STD4 had the greatest starter intake.

Effect of silage botanical composition on ruminal biohydrogenation and transfer of fatty acids to milk in dairy cows. By Adler et al., page 1135. Forage lipids have high proportions of polyunsaturated fatty acids, but extensive ruminal biohydrogenation and de novo synthesis give milk fat high proportions of saturated fatty acids, which are assumed to have negative effects on human health. However, milk is also a source of beneficial fatty acids, such as n-3 fatty acids and conjugated linoleic acids, with rumenic acid (C18:2 cis-9,trans-11) being the most important. In the present experiment, silage with a high proportion of red clover decreased ruminal biohydrogenation of fatty acids and increased milk fat proportion of $\alpha$-linolenic acid (C18:3n-3) and rumenic acid compared with pure grass silages.

Evaluation of the Brix refractometer to estimate immunoglobulin G concentration in bovine colostrum. By Quigley et al., page 1148. Accurate estimation of immunoglobulin concentration in colostrum is important to ensure the health of young dairy calves. Current methods are costly, slow, or not sufficiently accurate for routine use on the farm. Estimating total solids in colostrum using a Brix refractometer has been proposed as a method to estimate immunoglobulin content. Total solids estimated with Brix refractometers were highly correlated with colostrum immunoglobulin G content on 8 dairy farms. Our data support that the Brix refractometer is an acceptable, rapid, and low-cost method to estimate immunoglobulin $\mathrm{G}$ in bovine colostrum.

Effect of dietary phytate on phosphorus digestibility in dairy cows. By Ray et al., page 1156. Variability in phytate phosphorus $(\mathrm{P})$ digestion in ruminants may influence the availability of P. Improved understanding of ruminal and postruminal phytate $\mathrm{P}$ digestion and its effect on total $\mathrm{P}$ digestion in dairy cows would support more accurate prediction of $\mathrm{P}$ availability. This experiment with ruminally and ileally cannulated lactating cows investigated the effect of dietary phytate $\mathrm{P}$ on $\mathrm{P}$ digestion. Ruminal phytate $\mathrm{P}$ digestion was not negatively affected by dietary phytate $\mathrm{P}$ and phytate $\mathrm{P}$ was degraded in the large intestine. Phosphorus digestibility was influenced by dietary $\mathrm{P}$ content rather than form.

Preference and drinking behavior of lactating dairy cows offered water with different concentrations, valences, and sources of iron. By Gen- ther and Beede, page 1164. The upper tolerable concentration of iron in drinking water is $0.3 \mathrm{mg} / \mathrm{L}$. The objective was to determine if concentration, valence, or source (salt form) of iron ( $\mathrm{Fe}$ ) in water affected preference and drinking behavior. Lactating dairy cows did not prefer drinking water containing 0 versus $4 \mathrm{mg}$ of $\mathrm{Fe} / \mathrm{L}$, but drank less water with $8 \mathrm{mg}$ of total recoverable $\mathrm{Fe} / \mathrm{L}$. No differences in water intake or behavior were found for drinking water with ferrous versus ferric iron, or for source (salt) of iron. Our research indicates that lactating dairy cows tolerate concentrations up to $4 \mathrm{mg}$ of $\mathrm{Fe} / \mathrm{L}$ without reducing water intake.

Effect of flaxseed supplementation rate and processing on the production, fatty acid profile, and texture of milk, butter, and cheese. By Oeffner et al., page 117\%. To determine the optimal rate for extruded flaxseed supplementation, mid- to latelactation Holstein cows were fed $0,0.91,1.81$, or 2.72 $\mathrm{kg} / \mathrm{d}$ of extruded and $1.81 \mathrm{~kg} / \mathrm{d}$ of ground flaxseed. Increasing supplementation of extruded flaxseed gradually improved the fatty acid profile of milk, butter, or cheese without decreasing production parameters. Up to $2.72 \mathrm{~kg} / \mathrm{d}$ of extruded flaxseed can be fed to Holstein cows in mid to late lactation without decreasing production of milk and dairy products.

Effect of Origanum vulgare L. leaves on rumen fermentation, production, and milk fatty acid composition in lactating dairy cows. By Hristov et al., page 1189. This short-term experiment demonstrated that leaf material from Origanum vulgare L. can decrease enteric methane production in dairy cows within $8 \mathrm{~h}$ after feeding without negatively affecting milk production and improving feed efficiency. These data, however, have to be interpreted with caution because the results need to be replicated in long-term experiments.

Prevalence and risk factors for shedding of thermophilic Campylobacter in calves with and without diarrhea in Austrian dairy herds. By Klein et al., page 1203. Campylobacter is a pathogen and commensal of animals and is one of the most common cause of bacterial gastroenteritis in humans. Only limited information is available on the prevalence and importance of Campylobacter in calves. In this study, we evaluated the presence of Campylobacter in preweaned dairy calves. Campylobacter was shed by $15 \%$ of the calves and was present on $33 \%$ of the examined farms. Risk factors associated with the presence of Campylobacter were the presence of poultry on the farm, length of time the calf spent in the calving area, use of a shared feeding bucket, and the time of individual housing.

Assessment of archaeol as a molecular proxy for methane production in cattle. By McCartney et 
al., page 1211. This study demonstrated a significant relationship between fecal archaeol concentration (mg/ $\mathrm{kg}$ of dry matter) and enteric methane production (g/ $\mathrm{kg}$ of dry matter intake) measured using calorimetric chambers. Stage of lactation significantly affected this relationship, which may be due to higher dry matter intake in mid lactation. Some unaccounted for variation remains, which may be due to different activity levels of methanogens or selective retention of archaeol in the rumen.

Effects of different strategies for feeding supplements on milk production responses in cows grazing a restricted pasture allowance. By Auldist et al., page 1218. Cows consuming approimately $8 \mathrm{~kg}$ (dry matter, DM) of pasture/cow per day and 9 to 11 $\mathrm{kg} \mathrm{DM} / \mathrm{cow}$ per day of mixed ration containing alfalfa hay, barley grain, corn grain, and corn silage produced more milk fat and had improved energy-corrected milk responses than cows fed equivalent amounts of energy as barley grain in the parlor and pasture silage in the paddock, or as a simple mix of barley and pasture silage. Such strategies can be applied in grazing situations when pasture is limited.

Short communication: Diet-induced variations in milk fatty acid composition have minor effects on the estimated melting point of milk fat in cows, goats, and ewes: Insights from a metaanalysis. By Toral et al., page 1232. A meta-analysis of a database of milk fatty acid profiles from cows, goats, and sheep fed different dietary treatments revealed that the variability in the estimated melting point of milk fat is narrow compared with the variability of milk fatty acid percentages, which would support the hypothesis of a contribution of milk fat fluidity in the control of diet-induced variations in milk fat secretion. Furthermore, the calculated melting point of milk fatty acids was lower in sheep than in cows or goats, although the effects of dietary treatments on this parameter did not differ between ruminant species.

Genetic associations of test-day fat:protein ratio with milk yield, fertility, and udder health traits in Nordic Red cattle. By Negussie et al., page $123 \%$. This study evaluated the genetic association of an indicator trait of tissue energy balance - fat:protein ratio (FPR)—with milk yield, udder health, and fertility traits during lactation. The heritability of FPR ranged from 0.14 to 0.28 in first lactation. Fat:protein ratio had moderate genetic associations with interval fertility and udder health traits in early lactation. Moreover, FPR is a heritable trait that is easily available from routine milk recording schemes and it could be used as low-cost monitoring tool of health and fertility at the most critical phases of lactation. It could also be used in selection programs to improve robustness in dairy cows.

Short communication: Feed utilization and its associations with fertility and productive life in 11 commercial Pennsylvania tie-stall herds. By Vallimont et al., page 1251. Feed intake was measured on 970 cows in 11 Pennsylvania tie-stall herds, and cows with a high ratio of yield to intake had positive genetic correlations with days open (unfavorable) and productive life (favorable). Residual feed intake also had an unfavorable association with days open. Selection for feed utilization should take into account relationships with fertility and other functional traits to avoid unfavorable correlated responses as a result of selection for feed efficiency.

Short communication: A missense mutation in the PROP1 (prophet of Pit 1) gene affects male fertility and milk production traits in the US Holstein population. By Lan et al., page 1255. The objective of this study was to test the effects of prophet of Pit 1 (PROP1) gene on male fertility and milk production traits in Holstein dairy cattle. A missense mutation was found to be significantly associated with sire conception rate, productive life, protein yield, and net merit index.

Technical note: Characteristics and use of the Illumina BovineLD and GeneSeek Genomic Profiler low-density bead chips for genomic evaluation. By Wiggans et al., page 1258. The GeneSeek Genomic Profiler (GGP; Neogen Corp., Lincoln, NE) with 8,654 single nucleotide polymorphisms (SNP) and single-gene tests became available in February 2012. It was created using the add-on capability of the Illumina BovineLD BeadChip (Illumina Inc., San Diego, CA), which replaced the Bovine $3 \mathrm{~K}$ BeadChip in October 2011. The GGP contains 6,909 BovineLD SNP as well as additional SNP to improve imputation accuracy and provide single-gene tests. The GGP slightly improves accuracy of imputation to BovineSNP50 genotypes compared with the BovineLD chip. For September 2012 US genomic evaluations, 82,510 BovineLD and GGP genotypes were included. Reliability was 3 percentage points higher for evaluations using BovineLD and GGP genotypes compared with Bovine3K genotypes.

Technical note: Changes to herd cutoff date in conception rate evaluations. By Hutchison et al., page 1264. Fertility evaluations (service-sire conception rate, heifer conception rate, and cow conception rate) have similar opportunity edits. Edits for these traits were recently modified to use the most recent test date for each herd rather than a constant cutoff date. Using a herd-specific edit improved accuracy of the evalua- 
tions by accounting for differing opportunity to confirm pregnancy caused by discontinued testing or differences in herd testing schedules without having to discard a large number of the most recent inseminations.

Reproductive performance in a select sample of dairy herds. By Ferguson and Skidmore, page 1269. Herds selected for excellent reproductive performance were summarized in this paper. Excellent reproductive performance was associated with consistent control of insemination frequency, good conception rate, and timely pregnancy diagnosis. Different management programs, whether using estrus observation or timed artificial insemination programs, resulted in excellent reproductive efficiency.

Assessment of herd management on organic and conventional dairy farms in the United States. By Stiglbauer et al., page 1290. The organic dairy industry is one of the fastest growing agricultural sectors in the United States, making it an appropriate choice for current dairy research. The goal of our study was to evaluate management and herd health characteristics on organic and similarly sized conventional farms across the United States. A key finding was that organic farmers were much less likely to use support from external sources in regard to management and health advice compared with their conventional counterparts.

Improving bovine udder health: A national mastitis control program in the Netherlands. By
Lam et al., page 1301. In the Netherlands, a 5-yr mastitis control program was run, consisting of research and knowledge-transfer parts. The latter comprised 2 communication strategies: a central and a peripheral approach. The central approach used rational argumentation, including on-farm study group meetings. The peripheral approach tried to motivate farmers with nontechnical arguments, using mass media campaigns and other approaches. Over the course of the program, the clinical mastitis incidence rate decreased from 33.5 to 28.1 quarter cases per 100 cow-years at risk. For the Dutch dairy industry, investing $€ 8$ million in a national mastitis control program seemed to be cost effective.

Expanding the dairy herd in pasture-based systems: The role for sexed semen use on virgin heifers. By Hutchinson et al., page 1312. A model was developed to examine the effects of sexed semen use on rate of herd expansion and farm profitability in seasonal dairy production systems. Use of sexed semen in virgin heifers generated greater numbers of replacement heifers and facilitated faster rates of herd expansion, resulting in greater cumulative profit over a 15 -yr period compared with using conventional frozen-thawed semen. The greater rates of herd expansion, however, contributed to a more prolonged cash-flow deficit at times of low milk prices. 